\title{
Exendin-4 protects dopaminergic neurons by inhibition of microglial activation and matrix metalloproteinase- 3 expression in an animal model of Parkinson's disease
}

\author{
Sehee Kim, Minho Moon and Seungjoon Park \\ Department of Pharmacology, Biomedical Science Institute and Medical Research Center for Reactive Oxygen Species, Kyunghee University School of \\ Medicine, Seoul 130-071, Republic of Korea \\ (Correspondence should be addressed to S Park; Email: sjpark@khu.ac.kr)
}

\begin{abstract}
Exendin-4 is a naturally occurring more potent and stable analog of glucagon-like peptide-1 (GLP-1) that selectively binds at the GLP-1 receptor. It has been recently demonstrated that GLP-1 receptor stimulation preserves dopaminergic neurons in cellular and rodent models of Parkinson's disease (PD). 1-Methyl-4phenyl-1,2,3,6-tetrahydropyridine (MPTP) causes nigrostriatal dopaminergic neurotoxicity in rodents; previous studies suggest that activated microglia actively participate in the pathogenesis of PD neurodegeneration. However, the role of microglia in the neuroprotective properties of exendin-4 is still unknown. Here, we show that, in the mouse MPTP PD model, systemic administration of exendin-4 significantly attenuates the loss
\end{abstract}

of substantia nigra pars compacta $(\mathrm{SNpc})$ neurons and the striatal dopaminergic fibers. Exendin-4 prevents MPTP-induced microglial activation in the SNpc and striatum, and the expression of matrix metalloproteinase-3. In addition, exendin-4 also suppressed MPTP-induced expression of pro-inflammatory molecules and tumor necrosis factor $\alpha$ and interleukin-1 $\beta$. Our data indicate that exendin-4 may act as a survival factor for dopaminergic neurons by functioning as a microgliadeactivating factor and suggest that exendin-4 may be a valuable therapeutic agent for neurodegenerative diseases such as PD.

Journal of Endocrinology (2009) 202, 431-439

\section{Introduction}

Parkinson's disease (PD) is a chronic neurodegenerative disorder of muscle movement commonly characterized by a progressive loss of dopaminergic neurons in the substantia nigra pars compacta (SNpc) and the degeneration of projecting nerve fibers in the striatum (Langston 2002), which leads to tremors, muscular rigidity, bradykinesia, and postural and gait abnormalities. The presence of oxidative stress and inflammatory activity is one of the significant features of PD (Hirsch et al. 1998). Since microglia are a principal source of a variety of cytotoxic compounds, including reactive oxygen species, reactive nitrogen species, pro-inflammatory cytokines, and prostaglandins (Banati et al. 1993); microglial activation is known to play a key role in the pathogenesis of human PD and the 1-methyl-4phenyl-1,2,3,6-tetrahydropyridine (MPTP)-induced PD model (Vila et al. 2001). Several molecules released from stressed dopaminergic neurons, such as $\alpha$-synuclein, neuromelanin, and matrix metalloproteinase-3 (MMP-3), are found to be involved in microglial activation (Kim et al. 2005, 2007, Kim \& Joh 2006). Among these microglial activators, MMP-3 is known to be responsible for the activation of microglia and the release of NADPH oxidase-derived superoxide and nuclear factor- $\mathrm{\kappa B}$-mediated pro-inflammatory cytokines, and eventually exacerbates dopaminergic neurodegeneration. Moreover, it is known that dopaminergic neuronal cell death, microglial activation, and superoxide generation are dramatically attenuated in MMP-3 ${ }^{-1-}$ mice in MPTP-induced animal PD model (Kim et al. 2007), indicating a critical role of MMP-3 in dopaminergic neurodegeneration and neuroinflammation. Therefore, the molecules targeting pharmacological intervention of MMP-3 and microglial activation could be considered as plausible candidates for neuroprotective agents in PD.

Glucagon-like peptide-1 (GLP-1), an endogenous 30 -amino acid gut-brain peptide hormone, is synthesized from proglucagon-derived peptides in intestinal endocrine $\mathrm{L}$ cells and in selected neurons in the brain stem and hypothalamus (Doyle \& Egan 2001). GLP-1-(7-36)-amide is the major circulating biologically active form, with lesser amounts of the bioactive GLP-1-(7-37) form also detectable. The biological activities of GLP-1 include stimulation of glucose-dependent insulin secretion and insulin biosynthesis, inhibition of glucagon secretion and gastric emptying, and inhibition of food intake (Doyle \& Egan 2001). GLP-1 also has tropic effects, including pancreatic $\beta$-cell proliferation and 
neurogenesis as well as an inhibition of $\beta$-cell apoptosis (Brubaker \& Drucker 2004). However, the clinical application of GLP-1 is limited because of its short duration of action due to rapid degradation by dipeptidyl peptidase IV (Perfetti \& Merkel 2000). Exendin-4, a naturally occurring more potent and stable analog of GLP-1 that selectively binds to the GLP-1 receptor, is clinically used to treat type II diabetes mellitus (Perry \& Greig 2003). It is known that exendin-4 can pass through the blood-brain barrier (Kastin \& Akerstrom 2003) and exert central effects, including promotion of neurotropic or neuroprotective actions (Perry et al. 2002a,b) and enhancement of cognitive functions (During et al. 2003). Very recently, it has been reported that exendin-4 reverses key deficits in 6-hydroxydopamine or lipopolysaccharide (LPS) lesioned rats (Bertilsson et al. 2008, Harkavyi et al. 2008). Moreover, Li et al. (2009) reported that exendin-4 being administered in the lateral ventricle protected dopaminergic neurons against MPTP-induced degeneration, preserved dopamine levels, and improved motor function in the MPTP mice PD models. Taken together, these findings suggest that GLP-1 receptor stimulation in the central nervous system plays a critical role in regulating neuronal plasticity and cell survival. However, it is still unknown whether exendin-4 exerts its neuroprotective effect in MPTP-induced dopaminergic degeneration via inhibition of microglial activation. In the present study, we sought to determine whether systemic administration of exendin- 4 was able to protect nigrostriatal dopaminergic neurons from MPTP-induced toxicity. To address the protective mechanism of exendin-4, the effect of exendin-4 on microglial activation was investigated. Finally, we also examined whether MMP-3 participates in exendin-4-mediated neuroprotection.

\section{Materials and Methods}

\section{Animals, MPTP intoxication, and exendin-4 treatment}

Male C57Bl/6 mice (22-24 g, 8-weeks-old) were used in the present study. They were housed under controlled environmental conditions (12 h light: $12 \mathrm{~h}$ darkness) and acclimated for at least 1 week. Food and tap water were available ad libitum. Animals were randomly assigned to groups of five animals. The mice received four i.p. injections of MPTP $(20 \mathrm{mg} / \mathrm{kg})$ in saline at 2-h intervals in a single day and killed 1 or 7 days after the last injection as previously described (Liberatore et al. 1999, Wu et al. 2002). The control group received saline solution. All experiments were approved by the Kyunghee University Animal Care Committee and conducted according to the principles and procedures outlined in the NIH Guide for the Care and Use of Laboratory Animals. All efforts were made to minimize animal suffering and to reduce the number of mice used. To determine the neuroprotective effects of exendin-4 on MPTP-induced dopaminergic neuronal cell death in vivo, mice were given i.p. injections of exendin-4 $(10 \mu \mathrm{g} / \mathrm{kg}) 30 \mathrm{~min}$ prior to each MPTP injection for a total of four injections.

\section{Immunohistochemistry}

For immunohistochemical analysis, mice were anesthetized with xylazine and ketamine and then perfused transcardially with a freshly prepared solution of $4 \%$ paraformaldehyde in PBS. The brains were removed and post-fixed overnight in the same fixative before being immersed in a solution of $30 \%$ sucrose in PBS. Serial $30 \mu \mathrm{m}$-thick coronal tissue sections were cut using a microtome and immunostained as free-floating sections. Tissue sections were incubated overnight at $4{ }^{\circ} \mathrm{C}$ with one of the following primary antibodies: rabbit anti-tyrosine hydroxylase $(\mathrm{TH})$ antibody (1:2000, Chemicon, Temecula, CA, USA); rat anti-Mac-1 antibody (1:1000, Chemicon); goat anti-MMP-3 antibody (1:100, Santa Cruz Biotechnology, Santa Cruz, CA, USA); goat antitumor necrosis factor (TNF)- $\alpha$ antibody $(1: 200, \mathrm{R} \& \mathrm{D}$ Systems, Minneapolis, MN, USA); or goat anti-interleukin (IL) $-1 \beta$ antibody (1:100, R\&D Systems). The sections were incubated with appropriate biotinylated secondary antibody (1:200, Vector Laboratories, Burlingame, CA, USA) and then visualized using the avidin-biotin-peroxidase complex method with diaminobenzidine tetrahydrochloride as the chromogen. For fluorescent immunodetection of MMP-3, sections were washed and incubated with a secondary Cy2-donkey anti-goat $\operatorname{IgG}(1: 400)$. Sections were counterstained with DAPI and fluorescence was observed using a confocal microscope. All tissue sections from all experimental groups to be compared were processed at the same time using identical reagents.

\section{Histological quantification}

The total number of TH-stained SNpc neurons was manually counted in the right and left $\mathrm{SNpc}$ of every sixth section, throughout the entire extent of the SNpc, at 7 days after the last MPTP or saline injection using Stereo Investigator (MicroBrightField, Williston, VT, USA). The sections used for counting covered the entire rostrocaudal axis of the $\mathrm{SN}$ (AP, -2.7 to $-3.8 \mathrm{~mm}$ from bregma according to the atlas of Franklin \& Paxinos (2001)). This generally yielded 30-36 sections in a series. Analysis was performed using a computer-assisted image analysis system consisting of a Zeiss Axioscope-2 microscope equipped with a computercontrolled motorized stage, a video camera, and Stereo Investigator software. Each midbrain section was viewed at low power $(40 \times)$, and the SNpc was outlined using the set of anatomical landmarks defined previously (Nelson et al. 1996) and according to the atlas of Franklin \& Paxinos (2001). The number of TH-stained cells was then counted at high power $(400 \times)$. After all of the TH-stained neurons were counted, the estimate of the total number of neurons was calculated according to a previously described formula (West et al. 1991). 
The striatal density of $\mathrm{TH}$ immunoreactivity was determined using a Motic BA400 microscope and Motic Image Advanced 3.2 software (Motic Co., Ltd, Causeway Bay, Hong Kong, People's Republic of China). To determine striatal density of $\mathrm{TH}$ immunoreactivity, four striatal sections between bregma +0.98 and $+0.02 \mathrm{~mm}$ of each mouse were stained for $\mathrm{TH}$. Staining intensity of the striatum was determined using a Motic BA400 microscope and Motic Image Advanced 3.2 software (Motic Co., Ltd). A square frame of $900 \times 900 \mu \mathrm{m}$ was placed in the dorsal part of the striatum. A second square frame of $200 \times 200 \mu \mathrm{m}$ was placed in the region of the corpus callosum to measure background value. To control for variations in background illumination, the average of the background density readings from the corpus callosum was subtracted from the average of density readings of the striatum for each section.

\section{RNA extraction and RT-PCR}

Total RNA from the ventral midbrain was isolated using a previously reported standard procedure (Kamegai et al. 1998). The RNA was then precipitated with isopropanol, and the pellet was washed with 70\% ethanol, air dried, and dissolved in sterile diethyl pyrocarbonate (DEPC) water. The concentration and purity of RNA were determined with a NanoDrop spectrophotometer (NanoDrop Technologies, Inc., Wilmington, DE, USA). One microgram of total RNA was reverse transcribed using Superscript II reverse transcriptase (Life Technologies, Inc.) at $42{ }^{\circ} \mathrm{C}$ with random hexamer priming. An RNA control tube containing all of the reverse transcription reagents except reverse transcriptase was included as a negative control to monitor genomic DNA contamination. The resulting cDNA was amplified using the GeneAmp PCR System 2700 (Applied Biosystems, Foster City, CA, USA) or the LightCycler (Roche Diagnostics Ltd). Quantitative real-time PCR analysis was carried out with SYBR Green I and specific primers for TNF- $\alpha$ (NM_013693; sense, 5'-CCC AGA CCC TCA CAC TCA GAT-3'; antisense, 5'-AAC ACC CAT TCC CTT CAC AGA-3'), IL-1 $\beta$ (NM_008361; sense, 5'-ACC TTT TGA CAG TGA TGA GAA-3'; antisense, $5^{\prime}$-AAG ATG AAG GAA AAG AAG GTG-3'), proMMP-3 (NM_010809; sense, 5'-GAT CTC TTC ATT TTG GCC ATC TCT TC-3'; antisense, 5'-CTC CAG TAT TTG TCC TCT ACA AAG AA- $3^{\prime}$ ), and $\beta$-actin (NM_031144; sense, 5'-ATG GGT CAG AAG GAC TCC TAC G-3'; antisense, $5^{\prime}$-AGT GGT ACG ACC AGA GGC ATA C-3'). Details of the procedure for real-time PCR were as previously described (Park et al. 2004).

\section{Statistical analysis}

Data are presented as mean \pm s.E.M. ( $n=5 /$ group $)$. Each experiment was repeated at least twice, giving essentially identical results. Statistical analysis between groups was performed using one-way ANOVA and Holm-Sidak method for multiple comparisons using SigmaStat for Windows Version 3.10 (Systat Software, Inc., Point Richmond, CA, USA). $P<0.05$ was considered statistically significant.

\section{Results}

\section{Exendin-4 treatment attenuates MPTP-induced dopaminergic neurodegeneration}

As shown in Fig. 1A and B, repeated MPTP administration resulted in a more than $58 \%$ reduction in the number of $\mathrm{SNpc}$ dopaminergic neurons. The loss of TH-positive neurons was confirmed by Nissl staining (data not shown). i.p. administration of exendin-4 (500 nmol/ $\mathrm{kg})$ prevented the MPTP-induced loss of TH-positive SNpc neurons. Animals that received treatments of exendin-4 and MPTP showed increased viable $\mathrm{TH}$-positive neurons in the $\mathrm{SNpc}$ to $83 \%$ $(P<0 \cdot 01)$ of control after exendin-4 treatment. Exendin-4 alone did not significantly alter the number of TH-positive neurons. To determine whether exendin-4 can also prevent the loss of striatal dopaminergic fibers, we assessed the density of $\mathrm{TH}$ immunoreactivity in the striatum (Fig. 1A and C). Four injections of MPTP significantly reduced TH immunostaining in dopaminergic terminals by $37 \%$ compared with saline-treated control. Mice that received exendin-4 had striatal TH-positive fibers that were $95 \%$ of control, compared with only $63 \%$ in the MPTP (alone)-treated group.

\section{Effect of exendin-4 on MPTP-induced microgliosis}

To investigate whether the neuroprotective effect of exendin-4 is associated with the inhibition of the MPTP-induced microglial activation, we examined the expression of Mac-1, which is a specific marker for microglial activation (Gonzalez-Scarano \& Baltuch 1999). Immunohistochemical staining showed that MPTP administration resulted in a dramatic increase in the number of activated microglia and a change in morphology, consisting of a large cell body with poorly ramified short and thick processes, in both the SNpc and striatum $24 \mathrm{~h}$ after the last MPTP injection (Fig. 2 and Table 1), and these changes were still evident 7 days after the MPTP injections (data not shown). However, in MPTP-injected exendin-4-treated mice, $\mathrm{SNpc}$ and striatal Mac-1-positive cells were significantly decreased compared with the MPTP-injected vehicle-treated group.

\section{Effect of exendin-4 on MPTP-induced MMP-3 expression}

We performed immunohistochemistry and RT-PCR to investigate the effect of exendin-4 on MPTP-induced MMP-3 expression in the SNpc. Immunohistochemical analysis revealed that MMP-3 was barely detectable in saline-treated group whereas increased MMP-3 staining was observed in mice treated with MPTP (Fig. 3A). Using a TH antibody to detect dopaminergic neurons, we found that a 
A

Saline

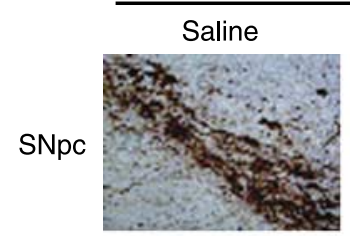

Exendin-4

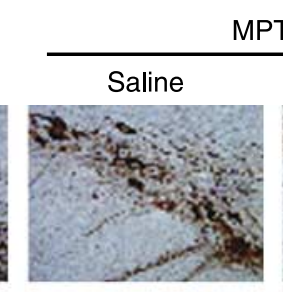

MPTP
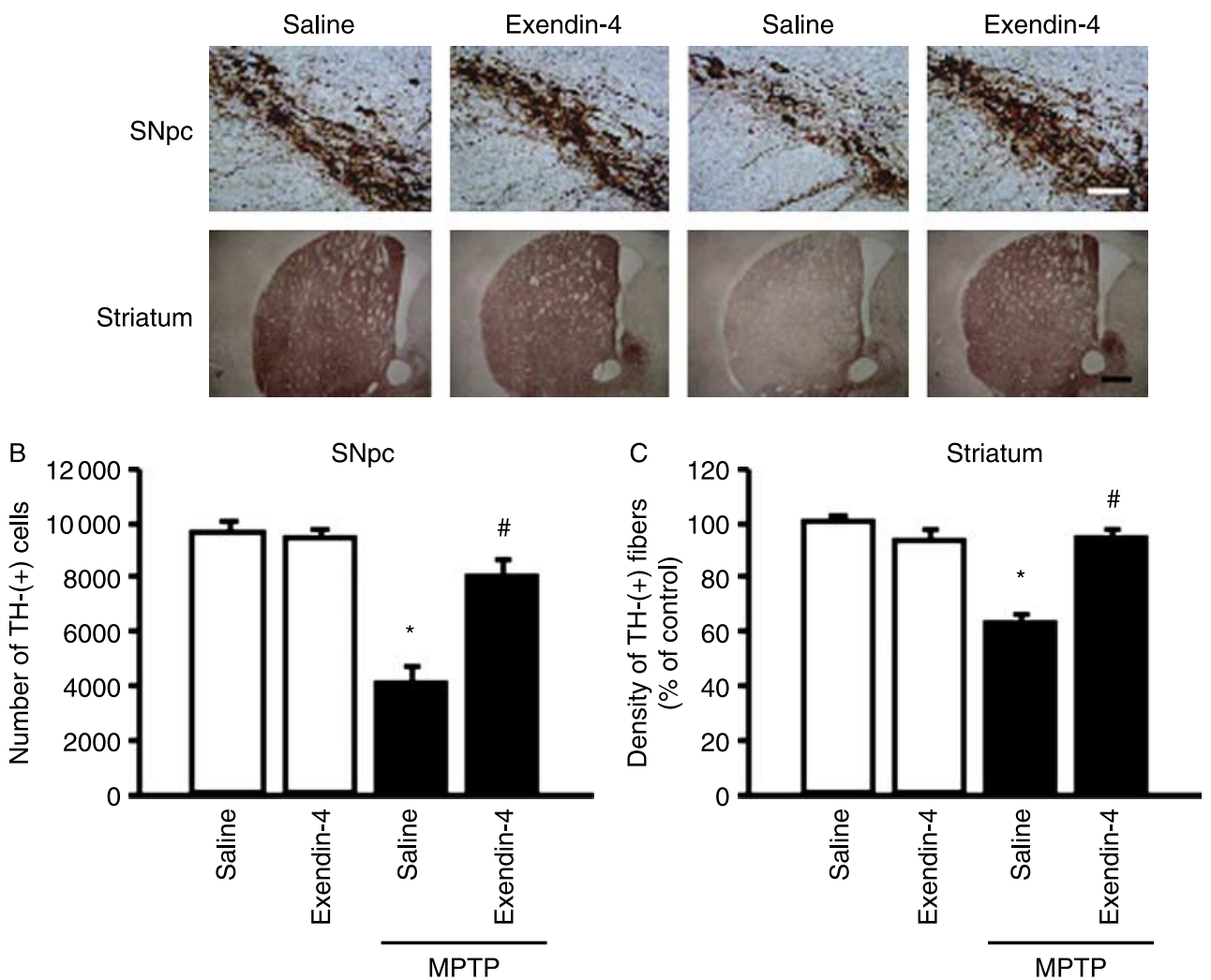

Figure 1 Neuroprotective effect of exendin-4 on 1-methyl-4-phenyl-1,2,3,6-tetrahydropyridine (MPTP)induced nigrostriatal dopaminrergic loss in vivo. C57Bl/6 mice were treated with four i.p. injections of MPTP in saline at 2-h intervals in a single day and sacrificed 7 days after the last injection. The control mice received saline solution. Either vehicle or exendin-4 was injected i.p. $30 \mathrm{~min}$ prior to each MPTP injection. (A) The number of substantia nigra pars compacta (SNpc) tyrosine hydroxylase (TH)-positive neurons and the density of striatal TH-positive fibers was determined by immunostaining coronal sections with anti-TH antibody. Representative photomicrographs of TH-positive SNpc neurons and striatal fibers are shown. Scale bars represent $100 \mu \mathrm{m}$ (for the SNpc) and $400 \mu \mathrm{m}$ (for the striatum). (B) and (C) Bar graphs representing the number of TH-positive SNpc neurons and striatal TH-positive optical density are shown. Values are mean \pm S.E.M. ( $n=5$ mice per group). ${ }^{*} P<0 \cdot 05$ versus saline-treated control; ${ }^{\sharp} P<0 \cdot 05$ versus MPTP-treated group. Full colour version of this figure available via http://dx.doi.org/10.1677/JOE-09-0132.

significant number of MMP-3-positive cells colocalized to dopaminergic neurons. Administration of exendin-4 significantly attenuated the up-regulation of MMP-3 by MPTP. These results indicate that exendin-4 is a potent inhibitor of MPTP-induced MMP-3 expression in experimental PD model in mice. RT-PCR analysis of proMMP-3 showed similar results (Fig. $3 \mathrm{~B}$ and $\mathrm{C}$ ).

\section{Effect of exendin-4 on the production of microglia-derived deleterious mediators}

It is well known that inflammation plays an important role in dopaminergic neurodegeneration in humans and the animal MPTP model of PD (Mogi et al. 1994, Hunot et al. 1996, Bessler et al. 1999, Dehmer et al. 2000, Nagatsu et al. 2000, Sriram et al. 2002). Given the fact that exendin-4 suppressed the MPTP-induced microglial response, we investigated if the expression of microglia-derived pro-inflammatory mediators in the midbrain is suppressed by exendin-4 treatment. As shown in Fig. 4A, MPTP injection resulted in a significant increase in TNF- $\alpha$ and IL- $1 \beta$ immunoreactivities in the SNpc. However, similar to the inhibition of microglial activation, exendin-4 treatment significantly suppressed the MPTP-induced increase of these pro-inflammatory cytokines. TNF- $\alpha$ and IL-1 $\beta$ mRNA levels assessed by RT-PCR analysis showed similar results (Fig. 4B and C).

\section{Discussion}

The present study demonstrated that systemic administration of exendin-4 protects nigrostriatal dopaminergic pathways from the Parkinsonian toxicity of MPTP in vivo by the inhibition of microglial activation and concomitant release 

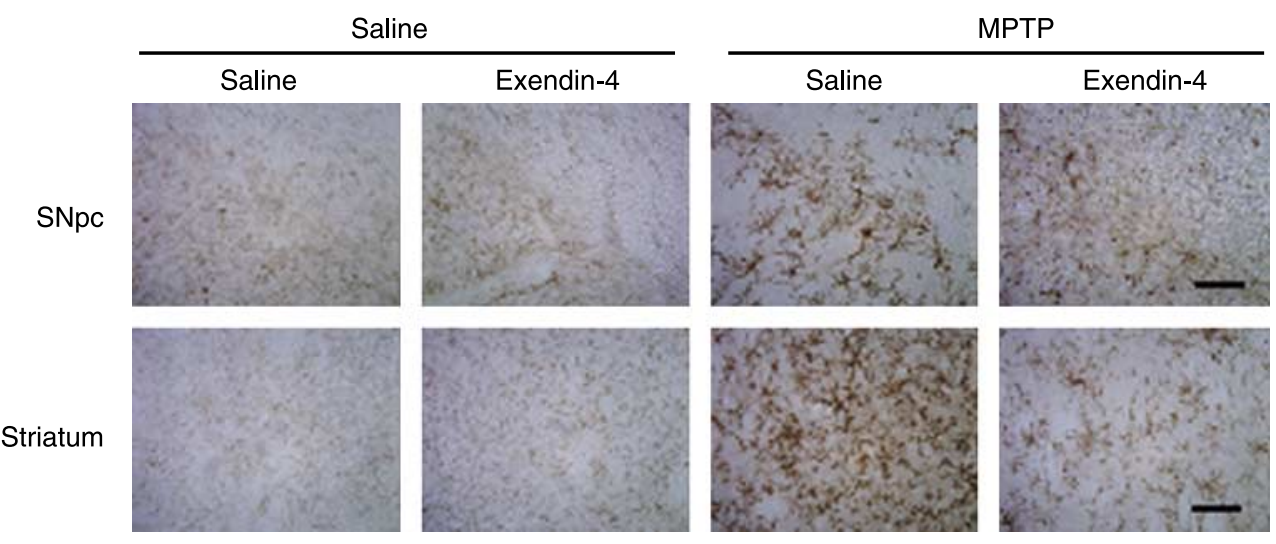

Figure 2 Exendin-4 prevents 1-methyl-4-phenyl-1,2,3,6-tetrahydropyridine (MPTP)-induced microglial activation. Mice were treated with MPTP or saline and sacrificed 1 day after the last MPTP injection. Animals were treated with exendin-4 i.p. $30 \mathrm{~min}$ prior to each MPTP injection. The number of activated microglia in the substantia nigra pars compacta (SNpc) and striatum was determined by immunostaining with antibody against Mac-1 in brain coronal sections. Representative photomicrographs of Mac-1 immunostaining in each group are shown. Scale bars represent $100 \mu \mathrm{m}$ (for the SNpc) and $50 \mu \mathrm{m}$ (for the striatum). Full colour version of this figure available via http://dx.doi.org/10.1677/JOE-09-0132.

of microglia-derived pro-inflammatory mediators. Exendin4-mediated suppression of reactive microgliosis seems to be associated with inhibition of microglial activator MMP-3 expression in the $\mathrm{SNpc}$ dopaminergic neurons.

It has been reported that GLP-1 receptor stimulation protects hippocampal neurons against various stimuli including amyloid- $\beta$ peptide-, $\mathrm{Fe}^{2+}$-, and glutamate-induced toxicity (During et al. 2003, Perry et al. 2003, Perry \& Greig 2005). Exendin-4 also has been reported to have protective effects in a number of classic cellular and animal models, such as stroke (Li et al. 2009), cholinergic ablation (Perry et al. 2002a), kainic acid-induced CA3 hippocampal loss (During et al. 2003), and peripheral neuropathy (Perry et al. 2007). In this study, we showed that dopaminergic neuron death in the SNpc was significantly reduced 1 week after MPTP administration in exendin-4-treated animals. We also found a similar protective effect on the loss of striatal dopaminergic nerve terminals exposed to MPTP. Given the fact that GLP-1 and exendin-4 can pass through the bloodbrain barrier after systemic administration (Kastin \& Akerstrom 2003) and exendin-4 given by this route has proven effective in alleviating peripheral neuropathy in rodents (Perry et al. 2007), these findings suggest that peripherally injected exendin-4 enters the brain parenchyma and exerts protective effects in the central nervous system. Our observation mirrors a recent report of Li et al. (2009), in which exendin-4 treatment provided complete protection of nigrostriatal dopaminergic neurons, dopamine and metabolite levels and ratios, and all behavioral abnormalities in a MPTP animal model of PD. Taken together, these findings provide evidence that exendin-4 may function as a survival factor for dopaminergic neurons by preventing the morphological abnormalities induced by MPTP neurotoxicity and offer a new perspective on the potential role of this peptide in Parkinson's-like neurodegenerative diseases.
The actions of GLP-1 and exendin- 4 are mediated through the GLP-1 receptor that is a member of the class B family of 7-transmembrane-spanning, heterotrimeric G proteincoupled receptors (Baggio \& Drucker 2007). In humans and rodents, GLP-1 receptors are widely expressed throughout the brain (Goke et al. 1995, Perry \& Greig 2003). GLP-1 activation of the Gas subunit of GLP-1 receptor leads to activation of adenylyl cyclase activity and increased production of intracellular cAMP (Li et al. 2003). Previous studies demonstrated that cAMP-mediated pathways are central to the antiapoptotic actions of GLP-1 in $\beta$-cells (Li et al. 2003, Perry \& Greig 2003, Baggio \& Drucker 2007), and the neuroprotective effects of cAMP-elevating agents are observed in many neuronal cells (D'Mello et al. 1993, Mena et al. 1995, Hanson et al. 1998). Other signaling pathways, such as PI3K and MAPK pathways, are also involved in GLP-1-mediated protective effects in neuronal cells (Perry et al. 2002b). However, the precise mechanisms by

Table 1 Effect of exendin-4 on 1-methyl-4-phenyl-1,2,3,6-tetrahydropyridine (MPTP)-induced microglial activation in the substantia nigra pars compacta (SNpc) and striatum. The number of Mac-1positive cells per $\mathrm{mm}^{2}$ with the change in morphology was determined as an index of microglial activation 1 day after MPTP administration. No Mac-1-immunostained cells with large cell bodies were found in saline-treated control and exendin-4-treated mice. Values are mean \pm S.E.M. $(n=5)$

\begin{tabular}{llll} 
& \multicolumn{1}{l}{ SNpc } & & Striatum \\
\cline { 2 - 2 } Saline & 0 & & 0 \\
Exendin-4 & 0 & & 0 \\
MPTP+ saline & $303 \cdot 3 \pm 9 \cdot 5$ & & $81 \cdot 8 \pm 5 \cdot 1$ \\
MPTP+ exendin-4 & $166 \cdot 3 \pm 5 \cdot 2^{*}$ & & $48 \cdot 5 \pm 11 \cdot 8^{*}$ \\
\hline
\end{tabular}

$* P<0.05$ versus MPTP-treated group. 

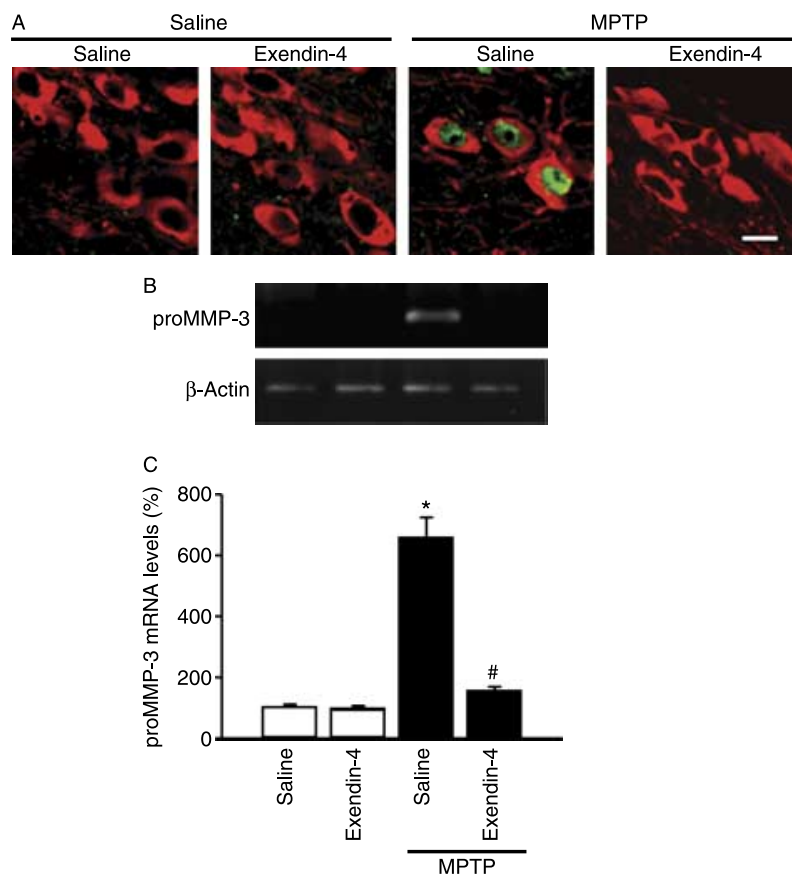

Figure 3 Exendin-4 attenuates 1-methyl-4-phenyl-1,2,3,6-tetrahydropyridine (MPTP)-induced matrix metalloproteinase-3 (MMP-3) expression in the substantia nigra pars compacta (SNpc). Mice were treated with either saline or MPTP at 2-h intervals in a single day. Animals were treated with exendin-4 i.p. 30 min prior to each MPTP injection. The mice were sacrificed 1 day after the last MPTP injection. (A) MMP-3 expression in the SNpc dopaminergic cells was determined by double fluorescent immunohistochemistry against MMP-3 (green) and TH (red). Representative photomicrographs of MMP-3 expression in $\mathrm{TH}$-positive SNpc neurons are shown. Scale bar represents $15 \mu \mathrm{m}$. (B) and (C) proMMP-3 mRNA levels were determined by RT-PCR (B) and real-time RT-PCR analysis (C). $\beta$-Actin was used as an internal control. Total RNA was extracted from the ventral midbrain. Values are mean \pm S.E.M. $(n=5$ mice per group). ${ }^{*} P<0.05$ versus saline-treated control; ${ }^{\#} P<0 \cdot 05$ versus MPTP-treated group. Full colour version of this figure available via http://dx.doi.org/10.1677/JOE-09-0132.

which exendin-4 protect dopaminergic neurons from MPTP-induced toxicity remain to be clarified.

Microglia, the resident immune cells in the brain (del Rio-Hortega 1993), can become overactivated by direct stimulation of microglia and neuronal damage and consequent reactive microgliosis (Block et al. 2007). Since activated microglial cells are important sources of proinflammatory cytokines such as TNF- $\alpha$, IL- $1 \beta$, and IFN- $\gamma$, reactive microgliosis could be an underlying mechanism of progressive neurodegeneration in PD (Kim \& Joh 2006). Previous studies reported that $\mathrm{SNpc}$ microglial activation was detected as early as $12 \mathrm{~h}$ and reached a maximum 1 day after MPTP injection; it subsided to control levels in about a week (Liberatore et al. 1999, Dehmer et al. 2000). Similar to these findings, we found that MPTP remarkably increased the number of activated microglia in both the $\mathrm{SNpc}$ and striatum $24 \mathrm{~h}$ after injection. Stimulatory signaling molecules, such as laminin, MMP-3, and $\boldsymbol{\alpha}$-synuclein, released from damaged dopaminergic neurons activate microglia to produce pro-inflammatory and neurotoxic factors, which are toxic to surrounding neurons, resulting in perpetuating cycle of neuronal death. This microglia-mediated self-perpetuating cycle of the neurotoxic activation of microglia in response to neuronal injury is one of the principal processes that urge progressive dopaminergic neurodegeneration (Liu 2006). Therefore, the neuroprotective agents targeting microglial activation may be able to break this vicious cycle and modulate the course of neurodegenerative processes. In fact, it has been demonstrated that several agents have neuroprotective effects due to their ability to suppress the microglial activation in several PD models (Liu et al. 2000, 2003, Wu et al. 2002, Delgado \& Ganea 2003). Therefore, we suggest that the ability of exendin-4 to prevent activation of microglial cells in both the $\mathrm{SNpc}$ and striatum plays an important role in its neuroprotective effect in this model. To our knowledge, this is the first report demonstrating the inhibition of MPTP-induced microglial activation by exendin-4.

It has been reported that MMP-3 plays critical roles in dopaminergic neurodegeneration in experimental models of PD both intracellularly, by mediating apoptosis of dopaminergic neurons, and extracellularly by triggering neuroinflammation (Kim et al. 2005, 2007, Choi et al. 2008). Specifically, active form of MMP-3 is released from dopaminergic neuronal cells that are under cellular stress and undergoing apoptosis and leads to microglial activation and production of pro-inflammatory and neurotoxic molecules in microglia and further enhances dopaminergic cell death (Kim et al. 2005, 2007). Furthermore, nigrostriatal dopaminergic neurodegeneration, microglial activation, and superoxide generation were substantially attenuated in MMP-3 ${ }^{-/-}$mice exposed to MPTP (Kim et al. 2007). In addition, it has been demonstrated that intracellular MMP-3 activity is altered in response to cellular stress due to the changes in gene expression and activation by cleavage of proMMP-3 (Choi et al. 2008). In the present study, we demonstrate for the first time that exendin-4 suppresses MPTP-induced MMP-3 expression in vivo. Since it is well known that MMP-3 plays a central role in dopaminergic neurodegeneration in $\mathrm{PD}$, inhibition of $\mathrm{MMP}-3$ expression by exendin-4 in early neuronal degeneration may be considered as promising therapeutic strategies to prevent progressive dopaminergic neuronal degeneration.

Activated microglia are a robust source of a variety of noxious compounds, such as superoxide, nitric oxide (NO), cytokines, and eicosanoids (Teismann et al. 2003, Teismann \& Schulz 2004). The levels of pro-inflammatory cytokines, such as TNF- $\alpha$ and IL-1 $\beta$, are increased in the SNpc glial cells of PD patients (Nagatsu et al. 2000). These cytokines may exert a direct effect on dopaminergic neurons by binding to specific cytokine receptors, then triggering intracellular death-related signaling pathways that leads to apoptosis or, alternatively, 
A

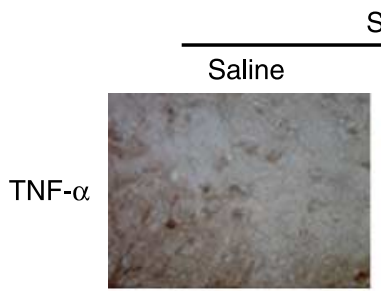

Saline
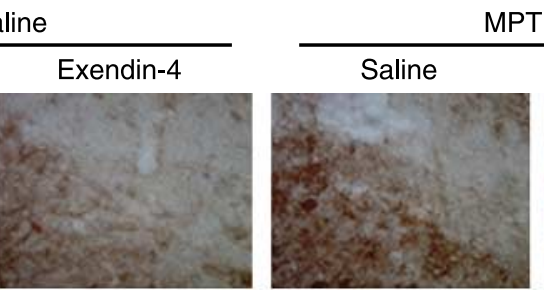

MPTP
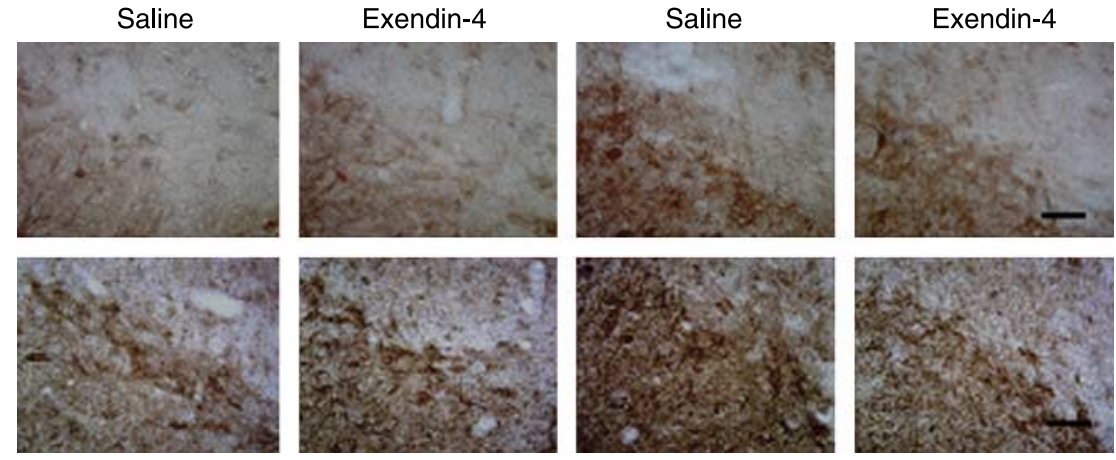

B

TNF- $\alpha$

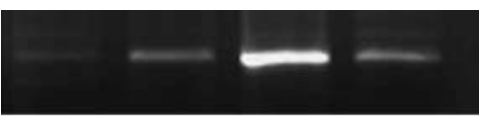

IL-1 $\beta$
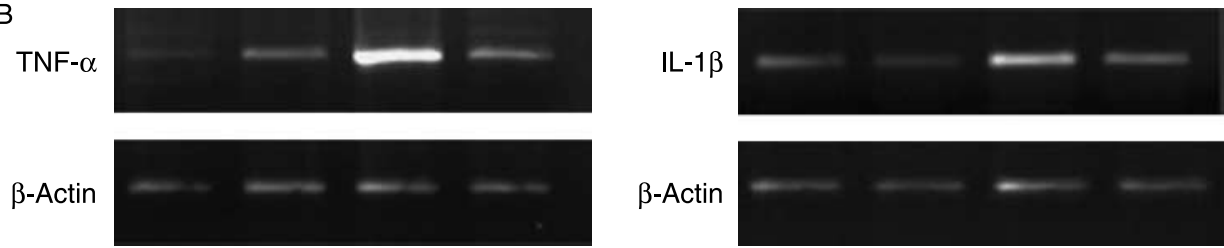

$\beta$-Actin
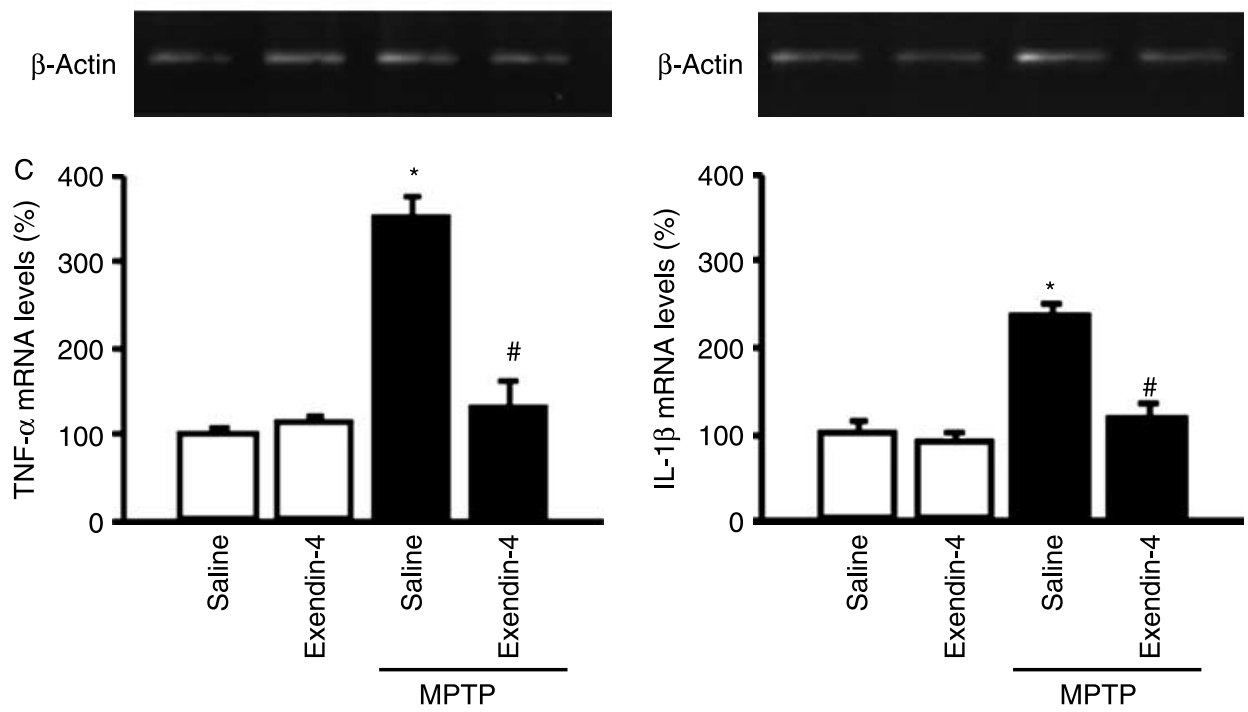

Figure 4 Exendin-4 prevents 1-methyl-4-phenyl-1,2,3,6-tetrahydropyridine (MPTP)-induced production of microglia-derived deleterious mediators in the ventral midbrain. Mice were treated with MPTP or saline injections and killed 1 day after the last MPTP injection. Animals were treated with exendin-4 i.p. $30 \mathrm{~min}$ prior to each MPTP injection. (A) TNF- $\alpha$ and IL-1 $\beta$ expression in the SNpc dopaminergic cells was determined by immunohistochemistry against TNF- $\alpha$ (upper panel) and IL-1 $\beta$ (lower panel). Representative photomicrographs of TNF- $\alpha$ and IL-1 $\beta$ expression in SNpc are shown. Scale bars represent $50 \mu \mathrm{m}$.

(B) and (C) TNF- $\alpha$ and IL-1 $\beta$ mRNA levels were determined by RT-PCR (B) and real-time RT-PCR analysis (C). Data represent mean \pm S.E.M. $(n=5) .{ }^{*} P<0 \cdot 05$ versus saline-treated control; ${ }^{\#} P<0 \cdot 05$ versus MPTP-treated group. Full colour version of this figure available via http://dx.doi.org/10.1677/JOE-09-0132.

indirectly by inducing the expression of inducible $\mathrm{NO}$ synthase (iNOS) within the microglia and cyclooxygenase-2 within the dopaminergic neurons, and then stimulating the formation of $\mathrm{NO}$ and superoxide respectively (Teismann $e t$ al. 2003). Microglia-derived NO-mediated nitrate stress plays a pivotal role in the pathogenesis of $\mathrm{PD}$, which is supported by the demonstration that MPTP-induced SNpc dopaminergic neuron loss and the production of ventral midbrain nitrotyrosine are attenuated in mice lacking the iNOS gene (Liberatore et al. 1999). NO can diffuse to neighboring dopaminergic neurons and react with superoxide to form the highly reactive tissue-damaging species peroxynitrite. In line with the concept that exendin-4 inhibits the MPTP-induced activation of microglia, we found that exendin- 4 attenuated the expression of TNF- $\alpha$ and IL- $1 \beta$ in the ventral midbrain. This inhibitory effect of exendin- 4 on TNF- $\alpha$ and IL- $1 \beta$ may contribute to alleviating MPTP-induced inflammatory damage. Supporting evidence for this observation is that GLP-1 could inhibit LPS-induced IL-1 $\beta$ mRNA expression and IL-1 $\gamma$ production in rat astrocytes (Iwai et al. 2006). 
Given the fact that the neuroprotective effect of exendin-4 is associated with the inhibition of MPTP-induced microglial activation, and the subsequent increase in TNF- $\alpha$ and IL-1 $\beta$, we suggest that exendin- 4 functions as a microgliadeactivating factor.

In summary, in agreement with the previous report of Li et al. (2009), we have demonstrated that peripheral administration of exendin-4 in the MPTP mouse model of PD attenuates nigrostriatal dopaminergic neuron loss. Moreover, we provide evidence for the first time that the neuroprotective effect of exendin-4 is mediated by the inhibition of microglial activation and the concomitant release of pro-inflammatory mediators. The inhibitory effect of exendin- 4 on microglial activation appears to be due to the inhibition of MMP-3 expression. These findings are significant because exendin- 4 can function as a neuroprotective agent and may have therapeutic potential for the treatment of PD and other neurodegenerative disorders where inflammatory responses play a major role.

\section{Declaration of interest}

The authors declare that there is no conflict of interest that could be perceived as prejudicing the impartiality of the research reported.

\section{Funding}

This work was supported by the Korea Research Foundation Grant funded by the Korean Government (MOEHRD, Basic Research Promotion Fund) (KRF-2008-E00503) and by the Korea Science and Engineering Foundation (KOSEF) grant (No. R13-2002-020-03001-0 (2007)).

\section{References}

Baggio LL \& Drucker DJ 2007 Biology of incretins: GLP-1 and GIP. Gastroenterology 132 2131-2157.

Banati RB, Gehrmann J, Schubert P \& Kreutzberg GW 1993 Cytotoxicity of microglia. Glia 7 111-118.

Bertilsson G, Patrone C, Zachrisson O, Andersson A, Dannaeus K, Heidrich J, Kortesmaa J, Mercer A, Nielsen E, Ronnholm H et al. 2008 Peptide hormone exendin- 4 stimulates subventricular zone neurogenesis in the adult rodent brain and induces recovery in an animal model of Parkinson's disease. Journal of Neuroscience Research 86 326-338.

Bessler H, Djaldetti R, Salman H, Bergman M \& Djaldetti M 1999 IL-1 $\beta$, IL-2, IL-6 and TNF- $\alpha$ production by peripheral blood mononuclear cells from patients with Parkinson's disease. Biomedicine and Pharmacotherapy 53 141-145.

Block ML, Zecca L \& Hong JS 2007 Microglia-mediated neurotoxicity: uncovering the molecular mechanisms. Nature Reviews. Neuroscience $\mathbf{8}$ $57-69$.

Brubaker PL \& Drucker DJ 2004 Minireview: glucagon-like peptides regulate cell proliferation and apoptosis in the pancreas, gut, and central nervous system. Endocrinology 145 2653-2659.

Choi DH, Kim EM, Son HJ, Joh TH, Kim YS, Kim D, Flint BM \& Hwang O 2008 A novel intracellular role of matrix metalloproteinase-3 during apoptosis of dopaminergic cells. Journal of Neurochemistry 106 405-415.

Dehmer T, Lindenau J, Haid S, Dichgans J \& Schulz JB 2000 Deficiency of inducible nitric oxide synthase protects against MPTP toxicity in vivo. Journal of Neurochemistry 74 2213-2216.
Delgado M \& Ganea D 2003 Neuroprotective effect of vasoactive intestinal peptide (VIP) in a mouse model of Parkinson's disease by blocking microglial activation. FASEB Journal 17 944-946.

D’Mello SR, Galli C, Ciotti T \& Calissano P 1993 Induction of apoptosis in cerebellar granule neurons by low potassium: inhibition of death by insulin-like growth factor I and cAMP. PNAS 90 10989-10993.

Doyle ME \& Egan JM 2001 Glucagon-like peptide-1. Recent Progress in Hormone Research 56 377-399.

During MJ, Cao L, Zuzga DS, Francis JS, Fitzsimons HL, Jiao X, Bland RJ, Klugmann M, Banks WA, Drucker DJ et al. 2003 Glucagon-like peptide-1 receptor is involved in learning and neuroprotection. Nature Medicine $\mathbf{9}$ 1173-1179.

Franklin KBJ \& Paxinos G 2001 In The Mouse Brain in Stereotaxic Coordinates, edn 2. Ed KBJ Franklin and G Paxinos. San Diego: Academic Press.

Goke R, Larsen PJ, Mikkelsen JD \& Sheikh SP 1995 Distribution of GLP-1 binding sites in the rat brain: evidence that exendin-4 is a ligand of brain GLP-1 binding sites. European Journal of Neuroscience 7 2294-2300.

Gonzalez-Scarano F \& Baltuch G 1999 Microglia as mediators of inflammatory and degenerative diseases. Annual Review of Neuroscience $\mathbf{2 2}$ $219-240$

Hanson MG Jr, Shen S, Wiemelt AP, McMorris FA \& Barres BA 1998 Cyclic AMP elevation is sufficient to promote the survival of spinal motor neurons in vitro. Journal of Neuroscience 18 7361-7371.

Harkavyi A, Abuirmeileh A, Lever R, Kingsbury AE, Biggs CS \& Whitton PS 2008 Glucagon-like peptide 1 receptor stimulation reverses key deficits in distinct rodent models of Parkinson's disease. Journal of Neuroinflammation 519

Hirsch EC, Hunot S, Damier P \& Faucheux B 1998 Glial cells and inflammation in Parkinson's disease: a role in neurodegeneration? Annals of Neurology 44 S115-S120.

Hunot S, Boissiere F, Faucheux B, Brugg B, Mouatt-Prigent A, Agid Y \& Hirsch EC 1996 Nitric oxide synthase and neuronal vulnerability in Parkinson's disease. Neuroscience 72 355-363.

Iwai T, Ito S, Tanimitsu K, Udagawa S \& Oka J 2006 Glucagon-like peptide-1 inhibits LPS-induced IL-1 $\beta$ production in cultured rat astrocytes. Neuroscience Research 55 352-360.

Kamegai J, Unterman TG, Frohman LA \& Kineman RD 1998 Hypothala$\mathrm{mic} /$ pituitary-axis of the spontaneous dwarf rat: autofeedback regulation of growth hormone $(\mathrm{GH})$ includes suppression of $\mathrm{GH}$ releasing-hormone receptor messenger ribonucleic acid. Endocrinology 139 3554-3560.

Kastin AJ \& Akerstrom V 2003 Entry of exendin-4 into brain is rapid but may be limited at high doses. International Journal of Obesity and Related Metabolic Disorders 27 313-318.

Kim YS \& Joh TH 2006 Microglia, major player in the brain inflammation: their roles in the pathogenesis of Parkinson's disease. Experimental and Molecular Medicine 38 333-347.

Kim YS, Kim SS, Cho JJ, Choi DH, Hwang O, Shin DH, Chun HS, Beal MF \& Joh TH 2005 Matrix metalloproteinase-3: a novel signaling proteinase from apoptotic neuronal cells that activates microglia. Journal of Neuroscience 25 3701-3711.

Kim YS, Choi DH, Block ML, Lorenzl S, Yang L, Kim YJ, Sugama S, Cho BP, Hwang O, Browne SE et al. 2007 A pivotal role of matrix metalloproteinase3 activity in dopaminergic neuronal degeneration via microglial activation. FASEB Journal 21 179-187.

Langston JW 2002 Parkinson's disease: current and future challenges. Neurotoxicology 23 443-450.

Li Y, Hansotia T, Yusta B, Ris F, Halban PA \& Drucker DJ 2003 Glucagonlike peptide-1 receptor signaling modulates beta cell apoptosis. Journal of Biological Chemistry 278 471-478.

Li Y, Perry T, Kindy MS, Harvey BK, Tweedie D, Holloway HW, Powers K, Shen H, Egan JM, Sambamurti K et al. 2009 GLP-1 receptor stimulation preserves primary cortical and dopaminergic neurons in cellular and rodent models of stroke and Parkinsonism. PNAS 106 1285-1290.

Liberatore GT, Jackson-Lewis V, Vukosavic S, Mandir AS, Vila M, McAuliffe WG, Dawson VL, Dawson TM \& Przedborski S 1999 Inducible nitric oxide synthase stimulates dopaminergic neurodegeneration in the MPTP model of Parkinson disease. Nature Medicine 5 1403-1409. 
Liu B 2006 Modulation of microglial pro-inflammatory and neurotoxic activity for the treatment of Parkinson's disease. AAPS Journal 8 E606-E621.

Liu B, Du L \& Hong JS 2000 Naloxone protects rat dopaminergic neurons against inflammatory damage through inhibition of microglia activation and superoxide generation. Journal of Pharmacology and Experimental Therapeutics 293 607-617.

Liu Y, Qin L, Li G, Zhang W, An L, Liu B \& Hong JS 2003 Dextromethorphan protects dopaminergic neurons against inflammationmediated degeneration through inhibition of microglial activation. Journal of Pharmacology and Experimental Therapeutics 305 212-218.

Mena MA, Casarejos MJ, Bonin A, Ramos JA \& Garcia YJ 1995 Effects of dibutyryl cyclic AMP and retinoic acid on the differentiation of dopamine neurons: prevention of cell death by dibutyryl cyclic AMP. Journal of Neurochemistry 65 2612-2620.

Mogi M, Harada M, Kondo T, Riederer P, Inagaki H, Minami M \& Nagatsu T 1994 Interleukin-1 $\beta$, interleukin-6, epidermal growth factor and transforming growth factor- $\alpha$ are elevated in the brain from parkinsonian patients. Neuroscience Letters 180 147-150.

Nagatsu T, Mogi M, Ichinose H \& Togari A 2000 Cytokines in Parkinson's disease. Journal of Neural Transmission 58 143-151.

Nelson EL, Liang CL, Sinton CM \& German DC 1996 Midbrain dopaminergic neurons in the mouse: computer-assisted mapping. Journal of Comparative Neurology 369 361-371.

Park S, Sohn S \& Kineman RD 2004 Fasting-induced changes in the hypothalamic-pituitary-GH axis in the absence of $\mathrm{GH}$ expression: lessons from the spontaneous dwarf rat. Journal of Endocrinology 180 369-378.

Perfetti R \& Merkel P 2000 Glucagon-like peptide-1: a major regulator of pancreatic $\beta$-cell function. European Journal of Endocrinology 143 717-725.

Perry T \& Greig NH 2003 The glucagon-like peptides: a double-edged therapeutic sword? Trends in Pharmacological Sciences 24 377-383.

Perry T \& Greig NH 2005 Enhancing central nervous system endogenous GLP-1 receptor pathways for intervention in Alzheimer's disease. Current Alzheimer Research 2 377-385.

Perry T, Haughey NJ, Mattson MP, Egan JM \& Greig NH 2002a Protection and reversal of excitotoxic neuronal damage by glucagon-like peptide-1 and exendin-4. Journal of Pharmacology and Experimental Therapeutics 302 881-888.

Perry T, Lahiri DK, Chen D, Zhou J, Shaw KT, Egan JM \& Greig NH $2002 b$ A novel neurotrophic property of glucagon-like peptide 1: a promoter of nerve growth factor-mediated differentiation in PC12 cells. Journal of Pharmacology and Experimental Therapeutics 300 958-966.
Perry T, Lahiri DK, Sambamurti K, Chen D, Mattson MP, Egan JM \& Greig NH 2003 Glucagon-like peptide-1 decreases endogenous amyloid-beta peptide (Abeta) levels and protects hippocampal neurons from death induced by Abeta and iron. Journal of Neuroscience Research 72 603-612.

Perry T, Holloway HW, Weerasuriya A, Mouton PR, Duffy K, Mattison JA \& Greig NH 2007 Evidence of GLP-1-mediated neuroprotection in an animal model of pyridoxine-induced peripheral sensory neuropathy. Experimental Neurology 203 293-301.

del Rio-Hortega P 1993 Art and artifice in the science of histology. 1933. Histopathology 22 515-525.

Sriram K, Matheson JM, Benkovic SA, Miller DB, Luster MI \& O'Callaghan JP 2002 Mice deficient in TNF receptors are protected against dopaminergic neurotoxicity: implications for Parkinson's disease. FASEB Journal 16 1474-1476.

Teismann P \& Schulz JB 2004 Cellular pathology of Parkinson's disease: astrocytes, microglia and inflammation. Cell and Tissue Research $\mathbf{3 1 8}$ 149-161.

Teismann P, Tieu K, Cohen O, Choi DK, Wu DC, Marks D, Vila M, Jackson-Lewis V \& Przedborski S 2003 Pathogenic role of glial cells in Parkinson's disease. Movement Disorders 18 121-129.

Vila M, Jackson-Lewis V, Guegan C, Wu DC, Teismann P, Choi DK, Tieu K \& Przedborski S 2001 The role of glial cells in Parkinson's disease. Current Opinion in Neurology 14 483-489.

West MJ, Slomianka L \& Gundersen HJ 1991 Unbiased stereological estimation of the total number of neurons in thesubdivisions of the rat hippocampus using the optical fractionator. Anatomical Record 231 482-497.

Wu DC, Jackson-Lewis V, Vila M, Tieu K, Teismann P, Vadseth C, Choi DK, Ischiropoulos H \& Przedborski S 2002 Blockade of microglial activation is neuroprotective in the 1-methyl-4-phenyl-1,2,3,6-tetrahydropyridine mouse model of Parkinson disease. Journal of Neuroscience 22 1763-1771.

Received in final form 24 June 2009

Accepted 29 June 2009

Made available online as an Accepted Preprint 29 June 2009 